\title{
Sweeping Surface Generated by a Class of Generalized Quasi-cubic Interpolation Spline*
}

\author{
Benyue $\mathrm{Su}^{1,2}$ and Jieqing Tan ${ }^{1}$ \\ ${ }^{1}$ Institute of Applied Mathematics, Hefei University of Technology, \\ Hefei 230009, China \\ ${ }^{2}$ Department of Mathematics, Anqing Teachers College, Anqing 246011, China \\ bysu1219@yahoo.com.cn
}

\begin{abstract}
In this paper we present a new method for the model of interpolation sweep surfaces by the $C^{2}$-continuous generalized quasicubic interpolation spline. Once given some key position, orientation and some points which are passed through by the spine and initial cross-section curves, the corresponding sweep surface can be constructed by the introduced spline function without calculating control points inversely as in the cases of B-spline and Bézier methods or solving equation system as in the case of cubic polynomial interpolation spline. A local control technique is also proposed for sweep surfaces using scaling function, which allows the user to change the shape of an object intuitively and effectively. On the basis of these results, some examples are given to show how the method is used to model some interesting surfaces.
\end{abstract}

\section{Introduction}

Sweeping is a powerful technique to generate surfaces in CAD/CAM, robotics motion design and NC machining, etc. There has been abundant research in the modeling of sweeping surfaces and their applications. $\mathrm{Hu}$ and Ling (2], 1996) considered the swept volume of a moving object which can be constructed from the envelope surfaces of its boundary. In this study, these envelope surfaces are the collections of the characteristic curves of the natural quadric surfaces. Wang and Joe $([13], 1997)$ presented sweep surface modeling by approximating a rotation minimizing frame. The advantages of this method lie in the robust computation and smoothness along the spine curves. Jüttler and Mäurer ([5], 1999) constructed rational representations of sweeping surfaces with the help

\footnotetext{
* This work was completed with the support by the National Natural Science Foundation of China under Grant No. 10171026 and No. 60473114, and in part by the Research Funds for Young Innovation Group, Education Department of Anhui Province under Grant No. 2005TD03, and the Anhui Provincial Natural Science Foundation under Grant No. 070416273X, and the Natural Science Foundation of Anhui Provincial Education Department under Grant No. 2006KJ252B, and the Funds for Science \& Technology Innovation of the Science \& Technology Department of Anqing City under Grant No. 2003-48.
} 
of the associated rational frames of $\mathrm{PH}$ cubic curves and presented sufficient conditions ensuring $G^{1}$ continuity of the sweeping surfaces. Schmidt and Wyvill (9, 2005) presented a technique for generating implicit sweep objects that support direct specification and manipulation of the surface with no topological limitations on the 2D sweep template. Seong, Kim et. al ([10, 2006) presented an efficient and robust algorithm for computing the perspective silhouette of the boundary of a general swept volume. In computer graphics, many advanced techniques using sweeping surfaces ([1], [3, 4]) have been applied to the deformation, NC simulation, motion traced and animation, including human body modeling and cartoon animation, etc. Yoon and Kim (14, 2006) proposed a approach to the freeform deformation(FFD) using sweeping surfaces, where a 3D object was approximated with sweep surfaces and it was easy to control shape

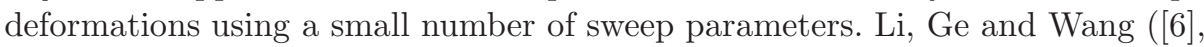
2006) introduced a sweeping function and applied it to the surface deformation and modeling, where the surface can be pulled or pushed along a trajectory curve.

In the process of constructing sweep surface, the hard work in modeling are to present simple objects and refine them towards the desired shapes, where the construction of the spine and across-section curves and the design of the moving frame (8]) are very important. Frenet frame, generalization translation frame and rotation-minimizing frame et. al (44, [5, 7, 13, 14]) can all be applied to solve these problem thoroughly.

In general, the spine curve can be presented by Bézier and B-spline methods. But they have many difficulties in calculating the data points conversely in order to interpolate given points. The main contribution of this paper is the development of a new method based on a class of generalized quasi-cubic interpolation spline. This approach has the following features:

- The spine and across-section curves are $C^{2}$ continuous and pass through some given points by the user without calculating the control points conversely as in the cases of B-spline and Bézier methods or solving equation system as in the case of cubic polynomial interpolation spline.

- A local control technique is proposed by the defined spline. It is implemented flexibly and effectively on the computer-human interaction.

- The moving frame is smoothness and can be established associated with the spine curve uniformly using our method.

The rest of this paper is organized as follows: A $C^{2}$-continuous generalized quasi-cubic interpolation spline is introduced in Sect. 2. We present a new method for the sweep surface modeling by the generalized quasi-cubic interpolation spline in Sect. 3. Some examples of shape modeling by the introduced method are given in Sect. 4. Finally, we conclude the paper in Sect. 5. 


\section{$2 C^{2}$-Continuous Generalized Quasi-cubic Interpolation Spline}

Definition 1. [11] Let $b_{0}, b_{1}, b_{2}, \cdots, b_{n+2},(n \geq 1)$, be given control points. Then a generalized quasi-cubic piecewise interpolation spline curve is defined to be

$$
p_{i}(t)=\sum_{j=0}^{3} B_{j, 3}(t) b_{i+j}, t \in[0,1], i=0,1, \cdots, n-1,
$$

where

$$
\begin{aligned}
& B_{0,3}(t)=\frac{1}{4}+\frac{t}{2}-\sin \frac{\pi}{2} t-\frac{1}{4} \cos \pi t+\frac{1}{2 \pi} \sin \pi t \\
& B_{1,3}(t)=-\frac{1}{4}+\frac{t}{2}+\cos \frac{\pi}{2} t+\frac{1}{4} \cos \pi t-\frac{1}{2 \pi} \sin \pi t \\
& B_{2,3}(t)=\frac{1}{4}-\frac{t}{2}+\sin \frac{\pi}{2} t-\frac{1}{4} \cos \pi t-\frac{1}{2 \pi} \sin \pi t \\
& B_{3,3}(t)=\frac{3}{4}-\frac{t}{2}-\cos \frac{\pi}{2} t+\frac{1}{4} \cos \pi t+\frac{1}{2 \pi} \sin \pi t
\end{aligned}
$$

From (2), we know that $B_{i, 3}(t),(i=0,1,2,3)$, possess properties similar to those of B-spline base functions except the positive property. Moreover, $p_{i}(t)$ interpolates the points $b_{i+1}$ and $b_{i+2}$. That is,

$$
p_{i}(0)=b_{i+1}, p_{i}(1)=b_{i+2},
$$

From (11) and (2), we can also get

$$
\begin{aligned}
& p_{i}^{\prime}(0)=\left(\frac{\pi}{2}-1\right)\left(b_{i+2}-b_{i}\right), \quad p_{i}^{\prime}(1)=\left(\frac{\pi}{2}-1\right)\left(b_{i+3}-b_{i+1}\right), \\
& p_{i}^{\prime \prime}(0)=\frac{\pi^{2}}{4}\left(b_{i}-2 b_{i+1}+b_{i+2}\right), p_{i}^{\prime \prime}(1)=\frac{\pi^{2}}{4}\left(b_{i+1}-2 b_{i+2}+b_{i+3}\right) .
\end{aligned}
$$

So

$$
p_{i}(1)=p_{i+1}(0), p_{i}^{(l)}(1)=p_{i+1}^{(l)}(0), l=1,2, i=0,1, \cdots, n-2 .
$$

Therefore, the continuity of the quasi-cubic piecewise interpolation spline curves is established up to second derivatives. Besides this property, the quasi-cubic piecewise interpolation spline curves also possess symmetry, geometric invariability and other properties, the details of these properties can be found in our another paper ([1] $)$.

\section{$3 \quad$ Sweep Surface Modeling}

Given a spine curve $P(t)$ in space and a cross-section curve $C(\theta)$, a sweep surface $W(t, \theta)$ can be generated by

$$
W(t, \theta)=P(t)+R(t)(s(t) \cdot C(\theta)),
$$

where $P(t)$ is a spine curve, $R(t)$ is an orthogonal matrix representing a moving frame along $P(t), s(t)$ is scaling function. Geometrically, a sweep surface $W(t, \theta)$ 
is generated by sweeping $C(\theta)$ along $P(t)$ with moving frame $R(t)$. cross-section curve $C(\theta)$ is in the $2 \mathrm{D}$ or $3 \mathrm{D}$ space which passes through the spine curve $P(t)$ during sweeping.

So the key problems in sweep surface generation are to construct the spine and cross-section curves $P(t), C(\theta)$ and to determine the moving frame $R(t)$.

Given a initial cross-sections $C_{j}(\theta)$ moving along a spine curve $P_{i}(t)$. Each given position is associated with a local transformation $R_{i}(t)$ on the $C_{j}(\theta)$. The sweep surface is generated by interpolating these key cross-sections $C_{i}(\theta)$ at these special positions by the user:

$$
\begin{aligned}
& W_{i, j}(t, \theta)=P_{i}(t)+R_{i}(t)\left(s_{i}(t) \cdot C_{j}(\theta)\right)
\end{aligned}
$$

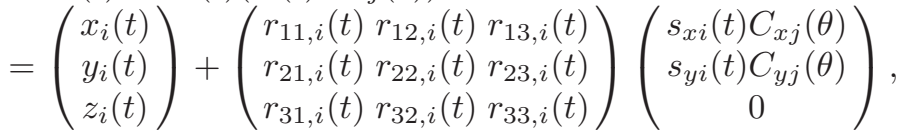

where the $s(t)$ is scaling function, which can be used to change the shapes of cross-sections to achieve local deformations.

\subsection{The Construction of Spine and Cross-Section Curves}

From the above discussions, we know that once some places that the crosssections will pass through are given, a spine curve can be constructed to interpolate these places (points) as follows:

$$
\begin{aligned}
& P_{i}(t)=\left(x_{i}(t), y_{i}(t), z_{i}(t)\right)^{T}=\sum_{j=0}^{3} B_{j, 3}(t) b_{i+j}, t \in[0,1], \\
& i=0,1, \cdots, n-1,
\end{aligned}
$$

where $b_{i}, i=0,1, \cdots, n+2,(n \geq 1)$, are given points (positions) by user, and $B_{j, 3}(t),(j=0,1,2,3)$, are generalized quasi-cubic piecewise interpolation spline base functions.

Similarly, if the explicit expression of cross-section curves are unknown in advance. But we know also the cross-section curves pass through some given points, then we can define the cross-section curves by

$$
\begin{aligned}
& C_{j}(\theta)=\left(C_{x j}(\theta), C_{y j}(\theta), 0\right)^{T}=\sum_{k=0}^{3} B_{k, 3}(\theta) q_{j+k}, \theta \in[0,1], \\
& j=0,1, \cdots, m-1,
\end{aligned}
$$

where $q_{j}, j=0,1, \cdots, m+2,,(m \geq 1)$, are given points (positions) by user.

In order to improve the flexibility and local deformation of the interpolation sweeping surfaces, we introduce scaling functions defined by

$$
\begin{aligned}
& s_{i}(t)=\left(s_{x i}(t), s_{y i}(t), 0\right)^{T}=\sum_{j=0}^{3} B_{j, 3}(t) s_{i+j}, t \in[0,1], \\
& i=0,1, \cdots, n-1,
\end{aligned}
$$

where $s_{i}=\left(\hat{s}_{i}, \tilde{s}_{i}, 0\right)^{T}, i=0,1, \cdots, n+2,(n \geq 1) . \hat{s}_{i}$ and $\tilde{s}_{i}$ are $n+3$ nonnegative real numbers respectively, which are called scaling factors. $B_{j, 3}(t),(j=0,1,2,3)$, are generalized quasi-cubic piecewise interpolation spline base functions. 


\subsection{The Moving Frame}

In order to interpolate their special orientations of key cross-sections, we can find a proper orthogonal matrix sequence $R(t)$ as a series of moving frame, such that $R(t)$ interpolate the given orthogonal matrices at the time $t=t_{i}$. Therefore, the interpolation problem lie in $R\left(t_{i}\right)=R_{i}$, where $R_{i}$ are the given orthogonal matrices at $t=t_{i}$.

For the given positions of the moving frames $\left(P_{i}, R x_{i}, R y_{i}, R z_{i}\right), i=0,1, \cdots$, $n-1$, we interpolate the translation parts $P_{i}$ by generalized quasi-cubic interpolation spline introduced in the above section, and we can also interpolate three orthogonal coordinates $\left(R x_{i}, R y_{i}, R z_{i}\right)$ homogeneously by the generalized quasi-cubic interpolation spline (Fig[1(a)). Namely,

$$
\begin{aligned}
& R_{i}(t)=\left(R x_{i}(t), R y_{i}(t), R z_{i}(t)\right)^{T}=\sum_{j=0}^{3} B_{j, 3}(t)\left(R x_{i+j}, R y_{i+j}, R z_{i+j}\right)^{T}, \\
& t \in[0,1], \quad i=0,1, \cdots, n-1
\end{aligned}
$$

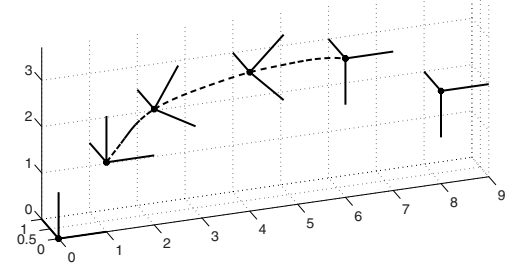

(a)

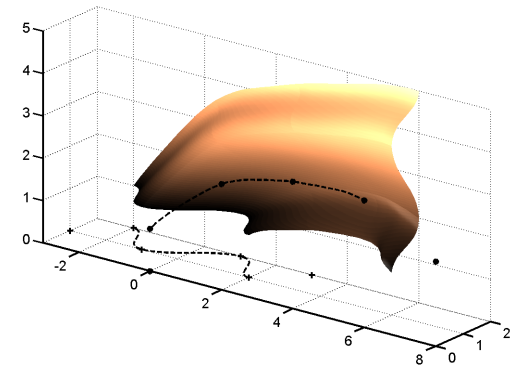

(b)

Fig. 1. (a) The moving frame on the different position, dashed line is spine curve. (b) The sweep surface associated with open cross-section curve.

Notes and Comments. Since $\left(R x_{i}(t), R y_{i}(t), R z_{i}(t)\right)$ defined by Eq. (11) usually does not form an accurate orthogonal coordinate system at $t \neq t_{i}$, we shall renew it by the Schmidt orthogonalization or an approximation of the orthogonal one with a controllable error. We can also convert the corresponding orthogonal matrices into the quaternion forms, then interpolate these quaternions by the (11) similarly, at last, the accurate orthogonal coordinate system can be obtained by the conversion inversely.

From the (7), (8) and (11), we know that for the fixed $\theta=\theta^{*}$,

$$
W_{i, j}\left(t, \theta^{*}\right)=\sum_{k=0}^{3} B_{k, 3}(t)\left(b_{i+k}+R_{i+k}\left(s_{i+k} \cdot q_{j}^{*}\right)\right),
$$

where $q_{j}^{*}=q_{j}\left(\theta^{*}\right)$, and for the fixed $t=t^{*}$, 


$$
W_{i, j}\left(t^{*}, \theta\right)=P_{i}^{*}+R_{i}^{*} \sum_{k=0}^{3} B_{k, 3}(t)\left(s_{i}^{*} \cdot q_{j+k}\right),
$$

where $P_{i}^{*}=P_{i}\left(t^{*}\right), R_{i}^{*}=R_{i}\left(t^{*}\right)$ and $s_{i}^{*}=s_{i}\left(t^{*}\right)$.

Since $q_{j}^{*}$ are constant vectors, we get that $W_{i, j}\left(t, \theta^{*}\right)$ are $C^{2}$-continuous and the points on curves $W_{i, j}\left(t, \theta^{*}\right)$ can be obtained by doing the transformation of stretching, rotation and translation on the point $q_{j}^{*}$.

The cross-section curves $W_{i, j}\left(t^{*}, \theta\right)$ at the $t=t^{*}$ can also be attained by the stretching, rotation and translation transformation on the initial cross-section curves $C_{j}(\theta)$.

Moveover, by computing the first and second partial derivatives of $W_{i, j}(t, \theta)$, we get

$$
\begin{aligned}
& \frac{\partial^{l}}{\partial t^{l}} W_{i, j}(t, \theta)=P_{i}^{(l)}(t)+\frac{d^{l}}{d t^{l}}\left(R_{i}(t)\left(s_{i}(t) \cdot C_{j}(\theta)\right)\right), \\
& \frac{\partial^{l}}{\partial \theta^{l}} W_{i, j}(t, \theta)=R_{i}(t)\left(s_{i}(t) \cdot C_{j}^{(l)}(\theta)\right),
\end{aligned}
$$

Then $W_{i, j}(t, \theta)$ are $C^{2}$-continuous with respect to $t$ and $\theta$ by the (5) and (14).

\section{The Modeling Examples}

Example 1. Given interpolating points of spine curve by $b_{0}=(0,0,1), b_{1}=$ $(0,0,1), b_{2}=(1,0,2.5), b_{3}=(2,0,3), b_{4}=(3,0,3), b_{5}=(4,0,2)$ and $b_{6}=(4,0,2)$. Suppose the initial cross-section curves pass through the points $\left(\cos \frac{(i-1) \pi}{6}\right.$, $\left.\sin \frac{(i-1) \pi}{6}\right), i=1,2, \cdots, 13$. The rotation angle at the four positions is $0, \pi / 3$, $\pi / 2$ and $2 \pi / 3$ respectively. Scaling factors are selected by $\hat{s}_{i}=\tilde{s}_{i} \equiv 1$. Then we get sweeping interpolation surface as in the Fig 2 (a) and Fig 3 .

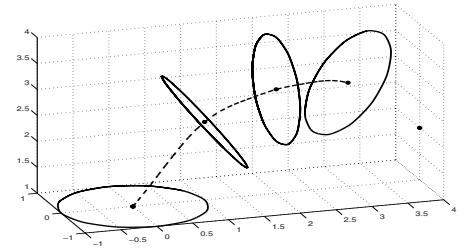

(a)

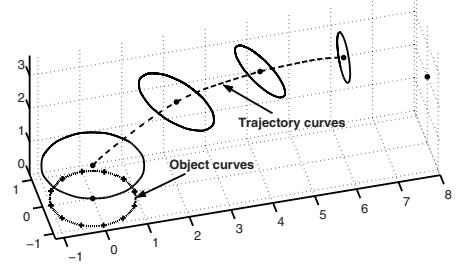

(b)

Fig. 2. The four key positions of cross-section curve during sweeping. (a) is the figure in example 1 and (b) is the figure in example 2

Example 2. Given interpolation points of spine curve by $b_{0}=(0,0,0), b_{1}=$ $(0,0,1), b_{2}=(2,0,2.5), b_{3}=(4,0,3), b_{4}=(6,0,3), b_{5}=(8,0,2)$ and $b_{6}=(8,0,2)$. The initial cross-section curve interpolates the points $\left(\cos \frac{(i-1) \pi}{6}, \sin \frac{(i-1) \pi}{6}\right), i=$ $1,2, \cdots, 13$. The rotation angle at the four positions is $0, \pi / 6, \pi / 4$ and $\pi / 2$ respectively. The scaling factors are chosen to be $\hat{s}_{i}=\tilde{s}_{i}=\{1.4,1.2,1,0.8,0.6,0.4,0.2\}$. Then we get sweeping interpolation surface as in the Fig 2 (b) and Fig 4 . 


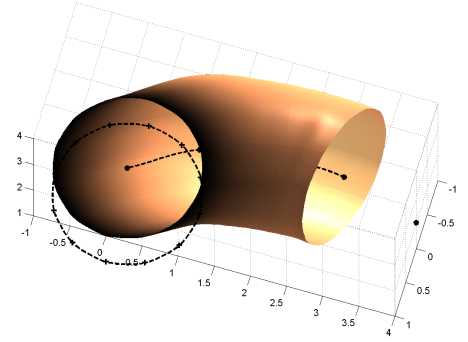

(a)

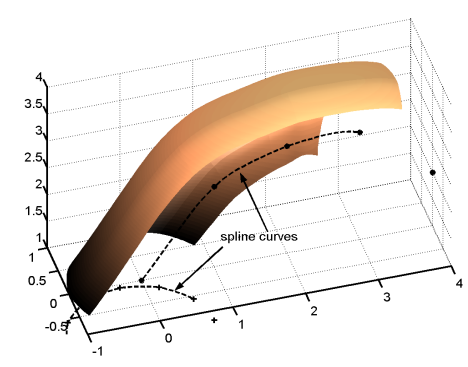

(b)

Fig. 3. The sweep surface modeling in example 1 (b) is the section plane of figure (a)

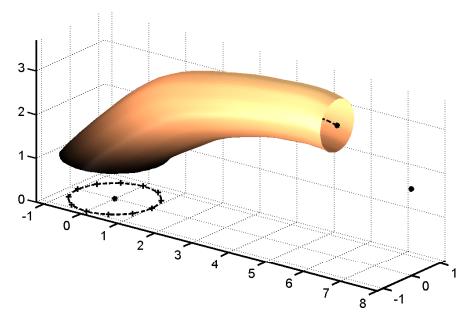

(a)

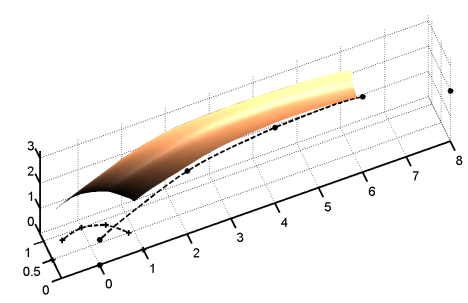

(b)

Fig. 4. The sweep surface modeling in example 2 (b) is the section plane of figure (a)

Example 3. The interpolation points of spine curve and rotation angles are adopted as in the example 2. The initial cross-section curve interpolates the points $q_{0}=(-3,1), q_{1}=(-2,2), q_{2}=(-1,1), q_{3}=(1,2), q_{4}=(2,1), q_{5}=(3,2)$. The scaling factors are chosen to be $\hat{s}_{i}=\tilde{s}_{i} \equiv 1$. Then we get the sweeping interpolation surface by open cross-section curve as in the Fig 1(b).

\section{Conclusions and Discussions}

As mentioned above, we have described a new method for constructing interpolation sweep surfaces by the $C^{2}$ continuous generalized quasi-cubic interpolation spline. Once given some key position and orientation and some points which are passed through by the spine and initial cross-section curves, we can construct corresponding sweep surface by the introduced spline function. We have also proposed a local control technique for sweep surfaces using scaling function, which allows the user to change the shape of an object intuitively and effectively.

Note that, in many other applications of sweep surface, the cross-section curves are sometimes defined on circular arcs or spherical surface, etc. Then we can construct the cross-section curves by the circular trigonometric Hermite interpolation spline introduced in our another paper ([12]).

On the other hand, in order to avoid a sharp acceleration of moving frame, we can use the chord length parametrization in the generalized quasi-cubic interpolation spline. 
In future work, we will investigate the real-time applications of the surface modeling based on the sweep method and interactive feasibility of controlling the shape of freeform $3 \mathrm{D}$ objects .

\section{References}

1. Du, S. J., Surmann, T., Webber, O., Weinert, K. : Formulating swept profiles for five-axis tool motions. International Journal of Machine Tools \& Manufacture 45 (2005) 849-861

2. Hu, Z. J., Ling, Z. K. : Swept volumes generated by the natural quadric surfaces. Comput. \& Graphics 20 (1996) 263-274

3. Hua, J., Qin, H. : Free form deformations via sketching and manipulating the scalar fields. In: Proc. of the ACM Symposium on Solid Modeling and Application, 2003, pp 328-333

4. Hyun, D.E., Yoon, S.H., Kim, M. S., Jüttler, B. : Modeling and deformation of arms and legs based on ellipsoidal sweeping. In: Proc. of the 11th Pacific Conference on Computer Graphics and Applications (PG 2003), 2003, pp 204-212

5. Jüttler, B., Mäurer C. : Cubic pythagorean hodograph spline curves and applications to sweep surface modeling. Computer-Aided Design 31 (1999) 73-83

6. Li, C. J., Ge, W.B., Wang, G.P. : Dynamic surface deformation and modeling using rubber sweepers. Lecture Notes in Computer Science 3942 (2006) 951-961

7. Ma, L.Z., Jiang, Z. D., Chan, Tony K.Y. : Interpolating and approximating moving frames using B-splines. In: Proc. of the 8th Pacific Conference on Computer Graphics and Applications (PG 2000), 2000, pp 154-164

8. Olver, P. J. : Moving frames. Journal of Symbolic Computation 36 (2003) 501-512

9. Schmidt, R., Wyvill, B. : Generalized sweep templates for implicit modeling. In: Proc. of the 3rd International Conference on Computer Graphics and Interactive Techniques in Australasia and South East Asia, 2005, pp 187-196

10. Seong, J. K., Kim, K. J., Kim, M. S., Elber, G. : Perspective silhouette of a general swept volume. The Visual Computer 22 (2006) 109-116

11. Su, B. Y., Tan, J. Q. : A family of quasi-cubic blended splines and applications. J. Zhejiang Univ. SCIENCE A 7 (2006) 1550-1560

12. Su, B. Y., Tan, J.Q. : Geometric modeling for interpolation surfaces based on blended coordinate system. Lecture Notes in Computer Science 4270 (2006) $222-231$

13. Wang, W. P., Joe, B. : Robust computation of the rotation minimizing frame for sweep surface modeling. Computer-Aided Design 23 (1997) 379-391

14. Yoon, S. H., Kim, M. S. : Sweep-based Freeform Deformations. Computer Graphics Forum (Eurographics 2006) 25 (2006) 487-496 